\title{
Conversion disorder, an importante slice of stroke mimics
}

D.M. Silva ${ }^{1}$, R. Martins ${ }^{1}$, R. Armindo ${ }^{2}$, C. Brito ${ }^{2}$, P. Vilela ${ }^{2}$.

${ }^{1}$ Centro Hospitalar Universitário do Algarve, Psychiatry department, Portimão, Portugal.

${ }^{2}$ Hospital Beatriz Ângelo, Neuroradiology department, Loures, Portugal

Purpose: An observational retrospective cohort study based on five years of experience aiming to characterize the group of patients with one of the most common clinical stroke mimics: conversion syndrome/psychiatric disorder.

Methods: From all the acute stroke protocols performed at Beatriz Ângelo Hospital, from November 2012 to October $2017(n=345)$, cases of probable conversive disorders (CD) were identified by two residents of psychiatry. Clinical, radiological and epidemiological data was reviewed and analyzed.
Conclusions: Conversion disorder is an important cause of stroke mimic. Some clinical and epidemiological data point to $C D$, not excluding ischemic stroke. In 5 years of experience of this service, CT Perfusion studies were normal in all patients with a final CD diagnosis. The correct application of a proper image control could be the fulcral point for the correct diagnosis, and this avoids the application of unnecessary therapies and with potential serious adverse effects.

(1)
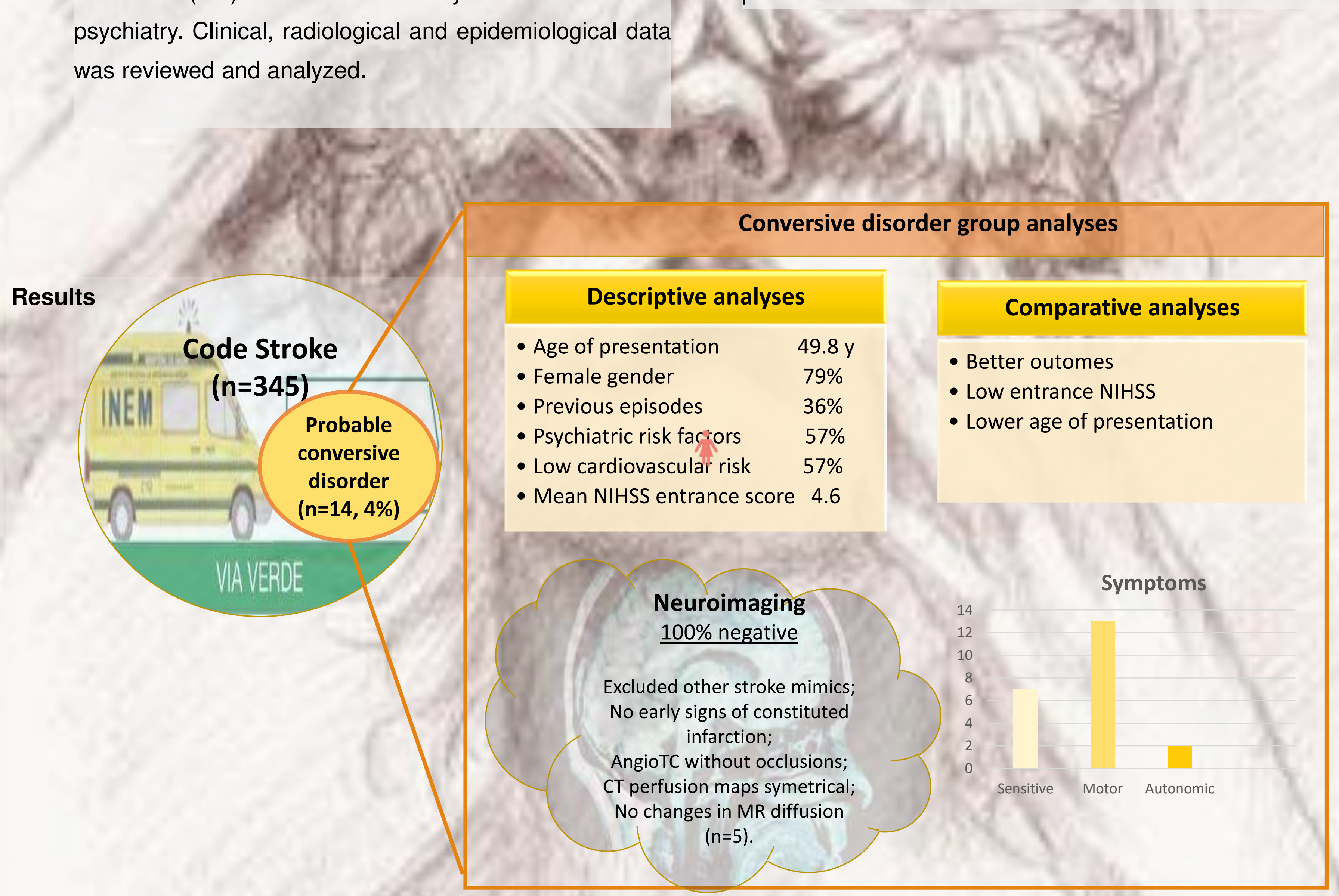

OUTCOMES

All patients had complete and spontaneous symptomatic remission; (93\% during the first $24 \mathrm{~h}$ )

The average hospitalization time was less than 3 days;

$71 \%$ of patients were referred to outpatient psychiatric follow-up

There were 2 episodes of recurrence of the condition.

Vilela P. Acute stroke differential diagnosis: Stroke mimics. Eur J Radiol. 2017;96:133-144; Perk J, De backer G, Gohlke H, et al. European Guidelines on cardiovascular disease prevention in clinical practice (version 2012). The Fifth Joint Task Force of the European Society of Cardiology and Other Societies on Cardiovascular Disease Prevention in Clinical Practice. Eur Heart J. 2012;33(13):1635-701. Wilkins SS, Bourke P, Salam A, et al. Functional Stroke Mimics: Incidence and Characteristics at a Primary Stroke Center in the Middle East. Psychosom Med. 2018;80(5):416-421. Caruso P, Manganotti P. Psychogenic Stroke Mimics and Thrombolysis: Ready to Take the Risk? Med Rep Case Stud 2016;1: 125. Chen Y, Bogosavljevic V, Leys D, Jovanovic D, Beslac-bumbasirevic L, Lucas C. Intravenous thrombolytic therapy in Stroke Mimics and Thrombolysis: Ready to Take the Risk? Med Rep Case Stud 2016;1: 125. Chen Y, Bogosavljevic V, Leys D, Jovanovic D, Beslac-bumbasirevic L, Lucas C. Intravenous thrombolytic therapy in
patients with stroke mimics: baseline characteristics and safety profile. Eur J Neurol. 2011;18(10):1246-50. Chernyshev OY, Martin-schild S, Albright KC, et al. Safety of tPA in stroke mimics and neuroimagingpatients with stroke mimics: baseline characteristics and safety profile. Eur J Neurol. 2011;18(10):1246-50. Chernyshev OY, Martin-schild S, Albright KC, et al. Safety of tPA in stroke mimics and neuroimaging-
negative cerebral ischemia. Neurology. 2010;74(17):1340-5. 